DEUTSCHES DANTE-JAHRBUCH 71. BAND 1996 


\section{DEUTSCHE DANTE-GESELLSCHAFT}

\section{VORSTAND}

Ehrenvorsitzender:

Prof. Dr. Dr. h.c. August BUCK, Georg-Voigt-Str. 5, 35039 Marburg/Lahn

Vorsitzender:

Prof. Dr. Bernhard KÖNIG, Romanisches Seminar der Universität Köln, AlbertusMagnus-Platz, 50923 Köln

Stellvertreter des Vorsitzenden:

Prof. Dr. Willi HIRDT, Romanisches Seminar der Rheinischen Friedrich-WilhelmsUniversität, Am Hof 1, 53113 Bonn

Geschäftsführer:

Herr Wolfgang HRADSKÝ, Finkensteig 13, 39110 Magdeburg

Herausgeber des Jahrbuchs:

Prof. Dr. Marcella RODDEWIG, Metzkauserstr. 21, 40625 Düsseldorf

Herausgeber des Mitteilungsblattes:

Frau Dr. Herma BASHIR-HECHT, Neubibergerstr. 15, 81737 München

Schriftführer:

Rechtsanwalt und Notar Karl-Christian KRETZSCHMAR, Theaterwall 45, 26122 Oldenburg

Bibliotheksverwalter:

Frau Dr. Herma BASHIR-HECHT, Neubibergerstr. 15, 81737 München

Prof. Dr. Georges GÜNTERT, Bahnweg 4, CH-8700 Küsnacht (ZH); Prof. Dr. Erika KANDUTH, Waltergasse 6-19, A-1040 Wien; Bibliotheksdirektor Lutz NEIDENBERG, Stadtbibliothek, Theaterplatz 2, 47798 Krefeld; Prof. Dr. Rainer STILLERS, Werner-Sombart-Str. 10, 78464 Konstanz.

\section{EHRENMITGLIEDER DER DEUTSCHEN DANTE-GESELLSCHAFT E.V.}

S. Kgl. Hoheit Markgraf Maria Emanuel von MEISSEN, Herzog zu Sachsen, Av. de Sully 102, CH-1814 La Tour de Peilz; Frau Eva FISCHER-RIES, Karl-LadenburgStr. 3, 68163 Mannheim; Frau Hella Ruth KAMPENDONK, Bernerstr. 7, 38106 Braunschweig; Prof. Dr. Francesco MAZZONI, Präsident der Società Dantesca Italiana, Piazza d'Azeglio 18, 1-50100 Firenze; Società Dantesca Italiana, Palagio dell'Arte della Lana, Via dell'Arte della Lana 1, I-50123 Firenze. 


\section{DEUTSCHES \\ DANTE-JAHRBUCH}

71. $B A N D$

HERAUSGEGEBEN IM AUFTRAG

DER DEUTSCHEN DANTE-GESELLSCHAFT E.V.

VON MARCELLA RODDEWIG

BÖHLAU VERLAG KÖLN WEIMAR WIEN 1996 
Jahresgabe der Deutschen Dante-Gesellschaft e.V. 1996. Die Mitglieder der Gesellschaft erhalten dieses Jahrbuch nach Überweisung der Mitgliederbeiträge gratis. Nur zu bestellen bei der Geschäftsführung der DDG, Herrn Wolfgang Hradský, Finkensteig 13, 39110 Magdeburg, Tel. 03917218246.

Anmeldung zur Mitgliedschaft durch einfache Erklärung bei der Geschäftsführung. Jahresbeitrag 60,- DM, für Studierende bis zum 30. Lebensjahr 30,- DM, für Ehepaare 90,- DM ab 1.1.1995. Die Konten der DDG sind:

a) Postgiroamt Köln Nr. 232273-507, BLZ 37010050

b) Commerzbank AG, Aachen, Konto-Nr. 500277900, BLZ 39040013

Manuskripte für das Jahrbuch wie Rezensionsexemplare für den Literaturbericht bitte an Prof. Dr. Marcella Roddewig, Metzkauserstr. 21, 40625 Düsseldorf, Tel. (0211) 289495 . Für unverlangte und ohne Rückporto eingesandte Manuskripte kann keine Gewähr übernommen werden.

Verlagsanschrift: Böhlau Verlag, GmbH \& Cie, Theodor-Heuss-Str. 76, 51149 Köln.

\section{VERZEICHNIS DER MITARBEITER DIESES BANDES}

Frau Dr. Barbara Bargagli Stoffi-Mühlethaler, Via di Fattucchia 18, I-50015 Grassina (FI)

Dr. Thomas Brückner, Olbrichstr. 7, 45138 Essen

Prof. Dr. Giovanni Cappello, Grise Piene 30, CH-2006 Neuchâtel

Herr Damian Dombrowski, Geiststraße 9, 48151 Münster

Dr. Axel Dunker, Fröbelstr. 12, 33604 Bielefeld

Prof. Dr. Remo Fasani, Rue Bachelin 37, CH-2000 Neuchâtel

Prof. Dr. Georges Güntert, Bahnweg 4, CH-8700 Küsnacht (ZH)

Prof. Dr. Bodo Guthmüller, Weintrautstr. 24, 35039 Marburg

Prof. Dr. Reinhard Klesczewski, Badeniastr. 12, 41564 Kaarst

Prof. Dr. Joachim Leeker, Erphostr. 24, 48145 Münster

Prof. Dr. Manfred Lentzen, Mersmannstiege 11, 48341 Altenberge

Prof. Dr. Siegfried Loewe, Guntherstr. 9/1/14, A-1150 Wien

Frau Dr. Lora Palladino, Postfach 111 303, 40513 Düsseldorf

Frau Dr. Monika Pauer, Metternichgasse 11, A-1030 Wien

Frau Dr. Valentina Pennacino, Via F. Aprile 4, I-16129 Genova

Frau Prof. Dr. Marcella Roddewig, Metzkauserstr. 21, 40625 Düsseldorf

Prof. Dr. Werner Ross, Über der Klause 1, 81545 München

Prof. Dr. Rainer Stillers, Werner-Sombart-Str. 10, 78464 Konstanz

Prof. Dr. Aldo Vallone, „Biblioteca Casa di Dante“, Via Siciliani, 5, I-73013 Galatina (Lecce)

Satz und Druck: Richarz Publikations Service, St. Augustin

Copyright (C) 1996 by Böhlau Verlag GmbH \& Cie, Köln

Printed in Germany

Einbandgestaltung: Herbert Meinke, Hamburg

ISSN 0070-444X 